CLINICAL STUDY

\title{
Bone mineral density and hip structural analysis in type 1 diabetic men
}

Tomasz Miazgowski, Sławomir Pynka, Marzena Noworyta-Ziętara, Barbara Krzyżanowska-Świniarska and Robert Pikul ${ }^{1}$

Department of Endocrinology, Hypertension and Metabolic Diseases Pomeranian Medical University, Szczecin, Poland and ${ }^{1}$ Department of Internal Diseases, Provincial Hospital, Szczecin, Poland

(Correspondence should be addressed to T Miazgowski; Email: miazgowski@interia.pl)

\begin{abstract}
Objective: The risk of non-vertebral fractures is increased in men with type 1 diabetes (DM1) but studies have shown only moderately decreased or normal bone mineral density (BMD) in these patients. No previous studies have evaluated hip strength and geometry indices in DM1 patients. This study was therefore designed to characterize associations between BMD, dual X-ray absorptiometry (DXA)-based hip strength indices, metabolic control, and DM1chronic complications.

Design and methods: The study was performed on 36 males aged 43.6 \pm 5.1 years with long-lasting DM1 and 36 healthy males matched for age, weight, and height. BMD in lumbar spine, total hip, upper and lower part of the femoral neck, hip axis length, cross-sectional area and moment of inertia (CSMI), and glycated hemoglobin (HbA1c) were measured.

Results: DM1 men had decreased spine BMD $(P<0.05)$ and normal total hip BMD in comparison with controls. Hip geometry and strength indices were comparable in both groups. However, M1 men had decreased CSMI and upper femur BMD but these differences did not reach statistical significance $(P=0.06)$. BMD changes and hip strength parameters did not correlate with HbA1c.

Conclusions: Middle-aged DM1 men have decreased lumbar spine BMD, normal hip BMD and normal hip strength indices. These changes are not influenced by metabolic control and presence of chronic microvascular complications.
\end{abstract}

European Journal of Endocrinology 156 123-127

\section{Introduction}

Osteoporosis represents a major public health problem because it is associated with increased mortality, morbidity, and health care costs. Decreased bone mineral density (BMD) assessed by dual X-ray absorptiometry (DXA) is a strong predictor of osteoporotic fractures (1). Many studies performed to date suggest that type 1 diabetes is associated with alternations in bone turnover, resulting in the development of osteopenia, in both adults $(2,3)$ and children $(4,5)$. The decrease of bone mass in diabetic patients is usually moderate and is not clearly associated with duration (5) or metabolic control of diabetes $(5,6)$, both in shortand long-term evaluation. Some of the earlier studies have suggested that diabetic osteopenia results in the increased fracture rate in type 1 diabetic women $(7,8)$. Recent findings have confirmed the increased fracture risk also in type 1 diabetic men. In a population-based cohort study with five repeated health surveys conducted on all residents of the Tromsø municipality, Ahmed et al. (9) have demonstrated that men with type
1 diabetes have an increased risk of non-vertebral (relative risk (RR) 3.1; 95\% CI 1.3-7.4) and hip fractures (RR 17.8; 95\% CI 5.6-56.8). These findings are consistent with the results of earlier (10) and recent (11) studies. BMD is not the sole factor influencing fracture occurrence. According to bioengineering principles, bone strength depends on (1) the mechanical properties of bone tissue, (2) geometrical arrangements, and (3) the magnitude, rate, and direction of force(s) applied to the bone $(1,12)$. Studies have demonstrated that the use of hip strength analysis (generated by newly developed DXA software) together with densitometric measurements of bone mass might improve the assessment of hip fracture risk better than BMD alone $(12,13)$. Several studies have shown that variation in geometrical measurements of the hip, such as increased hip axis length or femoral neck-shaft angle, was associated with increased fracture risk $(14,15)$. The hip strength analysis has so far mostly been used to study age-related changes in bone structure $(13,16)$, and to compare differences between genders $(13,15)$ and races (17). There have been no previous reports 
evaluating bone geometry and strength indices in type 1 diabetic patients. This study was therefore designed to characterize the associations between BMD measurements, DXA-based hip strength indices and metabolic control of type 1 diabetes.

\section{Subjects and methods}

\section{Study population}

The study was performed on 36 males aged $43.6 \pm 5.1$ years with long-standing type 1 diabetes, diagnosed according to recommendations of American Diabetes Association (18).

The long-term complications of diabetes, including diabetic retinopathy, nephropathy, and neuropathy were evaluated using routine diagnostic procedures (19). Metabolic control of diabetes was assessed by glycated hemoglobin (HbAlc) serum level by highperformance liquid chromatography. Strict metabolic control was defined by HbAlc serum level below $\%$ (19). The following exclusion criteria were applied: endocrine disorders other than DM1, any kind of malignancy, clinically significant (current or past) hepatic, gastro-intestinal, psychiatric or hematological disease, and renal injury, including overt nephropathy. Patients with current or past treatment with psychotropic drugs, corticosteroids, anticonvulsivants, statins, heparin, or antiosteoporotic drugs were not enrolled to the study. The control group consisted of 36 subjects recruited from local electoral registers. Invitation letters were sent to men matched for age to the study group. Subjects were considered healthy as they had no medical history, showed no abnormalities in the physical examination, declared no history of alcohol or narcotic abuse, and were not currently treated for any reason. There was no fracture history in both groups. The protocol and consent forms were approved by the institutional review board and all the participants gave a written informed consent.

\section{Anthropometric measurements}

Height and weight were measured without shoes. Height was measured with a single fixed stadiometer, and weight on a standard clinical balance. Body mass index was calculated as weight $(\mathrm{kg})$ divided by height (m) squared.

\section{Assessment of bone mineral density and femoral neck strength}

BMD and femoral neck strength indices were measured by DXA (GE Lunar Prodigy Advance, Madison, WI, USA; software enCORE version 8.8), using an automatic scan mode. BMD was measured in lumbar spine (L1-L4) and as a total hip region of interest (ROI) in the left femoral neck. BMD values were expressed in grams per centimeter squared as well as z-scores (number of standard deviations from mean BMD for normal, age-matched men) and $t$-scores (number of standard deviations from mean BMD for normal young men). The advanced hip assessment (AHA) software permits the calculation of bone geometry and bone tissue distribution within the femoral neck from a planar scan. With AHA it is possible to extract X-ray absorption data from the output image data file and from this to calculate the amount of bone mineral and its distribution. The femur ROI is placed automatically in the proximal part of the femoral neck. The software iteratively assesses all cross-sections in the femoral neck region of interest and identifies the plane with the minimum crosssectional moment of inertia $\left(\mathrm{CSMI}, \mathrm{cm}^{4}\right)$. CSMI is a measure of bone's resistance to bending forces.

For a given amount of bone within the femoral neck, the farther the bone is distributed from the center of mass within the cross-section, the more resistant it is. The CSMI assessed by DXA highly correlates with the CSMI measured directly on cadaver specimens and aluminum step wedge phantom (20). The crosssectional area $\left(\mathrm{CSA}, \mathrm{cm}^{2}\right)$ is a measure of the bone's resistance to axial forces and is proportional to bone mineral content in the defined cross-section of the femoral head. Besides this, the total femur BMD assessment, the AHA software allows for BMD measurements of upper (upper femur) and lower (lower femur) ROI of the femoral neck. Upper femur represents bone mineral content above the hip axis length line. This region is automatically determined by the software based upon the neck region of interest position and the calculated hip axis length line position. The hip axis length (HAL, cm) was evaluated as the linear distance from the pelvic rim to the lateral edge of the femur below greater trochanter and along the femoral neck axis. HAL was measured using the DXA ruler option. The reproducibility of the AHA was determined by five repeated scans performed in ten healthy men and calculation of the coefficients of variation $(\% \mathrm{CV})$. The CV values were: CSMI $2.8 \%$, CSA $2.2 \%$, HAL $0.5 \%$, and BMD 0.9\%.

\section{Statistical analysis}

Descriptive analyses were presented as mean \pm s.D., assuming $P<0.05$ as a significant difference between means. Differences between diabetics and controls were tested by Wilcoxon test or Mann-Whitney nonparametric $U$-test as appropriate. Linear correlation and regression or non-parametric regression analysis were used to test for relationship between BMD, bone strength indices, and other continuous variables. The chi-squared statistics was used to test associations between categorical variables. 


\section{Results}

Clinical characteristics of diabetic patients and healthy controls are summarized in Table 1. The control group was matched for age, height, weight, and BMI. Diabetic patients were characterized by long duration of the disease (range 19-39 years). In 76\% of the patients, eye changes in ophthalmoscopy were found, including 21 cases (55\%) with non-proliferative and 8 cases $(21 \%)$ with proliferative retinopathy. Microalbuminuria was found in $32 \%$ of patients, and tests for peripheral and/or autonomic neuropathy were positive in $29 \%$. Only seven patients (18\%) had HbAlc serum level below $7.0 \%$, a threshold value for a strict metabolic control of diabetes (19).

Type 1 diabetic men had significantly lower spine BMD and normal femur BMD in comparison with the controls (Table 2). The decrease of spine BMD ( $t$-score) between -1 and -2.5 s.D. was found in $41 \%$ of cases and in another $6 \%$ decrease of BMD was greater than 2.5 S.D. The prevalence of spine BMD decrease between -1 and -2.5 S.D. was significantly higher in the group of diabetic patients than in controls $(P<0.05)$. Femur BMD was decreased by $1-2.5$ s.D. in $11 \%$ of diabetic patients. Any significant correlation was found between level of HbAlc and spine BMD $(R=0.18 ; P=0.09)$ or femur BMD $(R=0.11 ; P=0.12)$. Also duration of diabetes did not correlate with spine $(R=-0.09$; $P=0.16)$ and femur BMD $(R=-0.11 ; P=0.12)$. The incidence of the BMD decrease was similar in patients with strict or poor metabolic control $(P=0.22)$. Similarly, we found no difference of BMD between patients with and without chronic vascular complications: retinopathy $(P=0.18)$, nephropathy $(P=0.08)$, and neuropathy $(P=0.10)$ in comparison with the controls. Patients with retinopathy had a similar BMD decrease in spine $(P=0.24)$ and femur $(P=0.13)$. The same finding was reported for nephropathy $(P=0.10$ and $P=0.18$ respectively) and neuropathy $(P=0.27$ and $P=0.32)$. In the control group no cases were with reduced spine or femur BMD by at least

Table 1 Clinical characteristics of type 1 diabetic patients and the controls.

\begin{tabular}{lcc}
\hline & $\begin{array}{c}\text { Diabetic } \\
\text { patients } \\
(n=38)\end{array}$ & $\begin{array}{c}\text { Controls } \\
(n=38)\end{array}$ \\
\hline Age (years) & $43.60 \pm 5.1$ & $44.09 \pm 6.1$ \\
Weight $(\mathrm{kg})$ & $74.14 \pm 8.2$ & $75.52 \pm 7.9$ \\
Height $(\mathrm{m})$ & $174.99 \pm 5.6$ & $175.23 \pm 5.8$ \\
BMl $\left(\mathrm{kg} / \mathrm{m}^{2}\right)$ & $24.54 \pm 2.8$ & $24.68 \pm 3.0$ \\
Smokers/non-smokers & $17 / 21$ & $12 / 26$ \\
Duration of diabetes (years) & $21.56 \pm 8.5$ & \\
Dosage of insulin (IU) & $46.2 \pm 4.4$ & \\
HbAlc (\%) & $7.56 \pm 0.9$ & \\
Retinopathy & 29 & \\
Nephropathy & 12 & \\
Neuropathy & 11 & \\
\hline
\end{tabular}

BMI, body mass index.
Table 2 Bone mineral density and hip strength indices in type 1 diabetic men and their respective age-matched controls.

\begin{tabular}{lrr}
\hline & $\begin{array}{c}\text { Diabetic } \\
\text { patients } \\
(n=38)\end{array}$ & \multicolumn{1}{c}{$\begin{array}{c}\text { Controls } \\
(n=38)\end{array}$} \\
\hline Spine $\left(\mathrm{g} / \mathrm{cm}^{2}\right)$ & $1.097 \pm 0.10$ & $1.118 \pm 0.14^{*}$ \\
Spine $(z$-score) & $-0.705 \pm 1.07$ & $-0.099 \pm 0.72^{\dagger}$ \\
Spine $(t$-score) & $-0.821 \pm 0.99$ & $-0.21 \pm 0.86^{\dagger}$ \\
Femur $\left(\mathrm{g} / \mathrm{cm}^{2}\right)$ & $0.907 \pm 0.11$ & $0.955 \pm 0.10$ \\
Femur $(z$-score $)$ & $-0.674 \pm 0.70$ & $-0.279 \pm 0.39$ \\
Femur $(t$-score & $-0.706 \pm 0.81$ & $-0.311 \pm 0.42$ \\
Upper femur $\left(\mathrm{g} / \mathrm{cm}^{2}\right)$ & $0.774 \pm 0.12$ & $0.808 \pm 0.12$ \\
Upper femur $(z$-score $)$ & $-0.443 \pm 0.85$ & $-0.111 \pm 0.53$ \\
Upper femur $(t$-score) & $-0.493 \pm 0.91$ & $-0.119 \pm 0.49$ \\
Lower femur $\left(\mathrm{g} / \mathrm{cm}^{2}\right)$ & $1.024 \pm 0.16$ & $1.084 \pm 0.10$ \\
Trochanter $\left(\mathrm{g} / \mathrm{cm}^{2}\right)$ & $0.852 \pm 0.09$ & $0.870 \pm 0.09$ \\
CSMI $\left(\mathrm{cm}^{4}\right)$ & $1.649 \pm 0.48$ & $1.791 \pm 0.39$ \\
CSA $\left(\mathrm{cm}^{2}\right)$ & $1.601 \pm 0.24$ & $1.652 \pm 0.29$ \\
HAL $\left(\mathrm{cm}^{2}\right)$ & $10.282 \pm 0.33$ & $10.512 \pm 0.49$ \\
\hline
\end{tabular}

CSMI, cross-sectional moment of inertia; CSA, cross-sectional area; HAL, hip axis length. ${ }^{*} P<0.05,{ }^{\dagger} P<0.01$ for the comparison between diabetic patients and controls.

1 s.D. to fulfill the World Health Organisation diagnostic criteria for osteopenia and osteoporosis (21). Analysis of AHA measurements showed no significant difference between the groups. In comparison with healthy individuals, diabetic men had similar CSA and decreased CSMI and upper femur BMD, but these differences were not significant $(P=0.06)$. Smoking did not influence BMD or hip strength indices both in diabetics and controls. Correlation among all analyzed variables was on average similar in both groups. Correlation coefficients for combined groups of type 1 diabetic men and healthy controls are given in Table 3. When BMD and the other continuous variables were analyzed in both groups in a multivariate regression model, weight, height, and femur BMD accounted for $67 \%$ of the variance of CSMI. Additionally, in diabetic patients, duration of the disease also influenced this variance and was negatively correlated with CSMI $(R=-0.34 ; P<0.05)$.

\section{Discussion}

The results of present study indicate that in middle-aged type 1 diabetic men lumbar spine BMD is decreased and femur BMD is normal, when compared with healthy men, matched for age, weight, and height. In majority of earlier studies performed on type 1 diabetic population, both lumbar spine and hip BMD was decreased moderately. However, some studies reported normal hip BMD $(22,23)$, and normal $(24)$ or even increased spine BMD (25). Nevertheless, there is agreement that approximately $40-50 \%$ diabetic patients have decreased BMD and this decrease is independent of age, diabetes duration, presence of chronic microvascular complications, and the onset of 
Table 3 Correlations between anthropometric and densitometric indices, both groups combined.

\begin{tabular}{|c|c|c|c|c|c|c|c|c|}
\hline & Age & Weight & Height & Femur BMD & Spine BMD & CSA & CSMI & HAL \\
\hline Age & - & & & & & & & \\
\hline Weight & 0.11 & - & & & & & & \\
\hline Height & 0.02 & $0.43^{*}$ & - & & & & & \\
\hline Femur BMD & -0.18 & $0.68^{*}$ & 0.20 & - & & & & \\
\hline Spine BMD & 0.11 & 0.19 & 0.21 & $0.49^{\star}$ & - & & & \\
\hline CSA & 0.09 & $0.59^{\star}$ & $0.41^{*}$ & $0.62^{\star}$ & 0.22 & - & & \\
\hline CSMI & -0.02 & $0.51^{*}$ & $0.59^{\star}$ & $0.83^{\dagger}$ & -0.15 & $0.62^{*}$ & - & \\
\hline $\mathrm{HAL}$ & -0.01 & 0.22 & $0.43^{*}$ & 0.12 & -0.14 & -0.08 & 0.10 & - \\
\hline
\end{tabular}

BMD, bone mineral density; CSA, cross-sectional area; CSMI, cross-sectional moment of inertia; HAL, hip axis length. ${ }^{\star} P<0.01,{ }^{\dagger} P<0.001$.

diabetes $(5,6,26)$, similar to our results. Bone loss was found even in children, at the time of diagnosis $(6,27)$. This may suggest that bone loss in type 1 diabetic men may not be viewed as part of constellation of known chronic, microvascular complications of diabetes (retinopathy, nephropathy, and neuropathy) but rather two distinct outcomes related to diabetes progression. We also found no association between BMD and HbAlc, suggesting that diabetes control does not play a major role in the pathogenesis of bone loss in type 1 diabetes. However, this finding is limited by the fact that HbAlc result is only reflective of short-term glycemic control (19), and it would not be expected to reflect cumulative bone damage measured by BMD. On the other hand, if the relationship between diabetes and bone loss was only related to hyperglycemia, one would expect a similar incidence of osteoporosis in patients with type 1 and those with type 2 diabetes, but osteoporosis is rather an uncommon feature of type 2 diabetes (28). There may be differences between two types of diabetes other than glycemic control that affect BMD decrease. Several possible factors have been investigated but the actual mechanisms involved in lower BMD in type 1 diabetes are not known. Increasing evidence suggests that insulin deficiency in type 1 diabetes may interfere with new bone formation, possibly due to defective function of osteoblasts. The impaired recruitment and function of osteoblasts might be an effect of increased osmolarity in poorly controlled diabetes (29), lower levels of insulin-like growth factor I (30), or accumulation of advanced glycation end-products in the type I collagen matrix (31). Earlier theory on the link between diabetic osteopenia and bone marrow microangiopathy (32) has not been confirmed in other studies. Our results confirm the findings from previous studies that bone mass deficiency observed in type 1 diabetic men is relatively moderate. It seems rather unlikely that such slight BMD decrease might contribute to nearly 18 -fold relative risk of hip fractures reported in the recent study (9). Moreover, BMD is not the sole factor influencing whether or not a fracture will occur. We addressed this issue for the evaluation of bone strength. Using AHA software, we demonstrated for the first time that in type 1 diabetic men, the hip geometry and strength indices do not differ from those measured in age-matched healthy controls. In comparison with healthy individuals, diabetic men had similar CSA and decreased CSMI and upper femur BMD, but these differences did not reach the statistical significance. The observed trend to the upper femur BMD decrease seems to be relevant, since the upper portion of the femoral neck has been reported to be a sensitive predictor of neck fractures. Studies have demonstrated that femoral fractures are commonly initiated in the upper neck region, and thickness as well as porosity of the bone in the upper neck region is believed to be critical to maintain femoral strength (33). Hip geometry and strength was not influenced by the duration of the disease, the metabolic control, and the presence of chronic complications of diabetes. However, the results of this study should be viewed cautiously given that this is relatively small cross-sectional study and the sample size was modest, which could limit the statistical power to detect a modest association. Larger longitudinal studies assessing the association of bone strength, BMD, and longterm metabolic control of diabetes are needed. Some potential limitations should be considered in interpreting the results of this study. BMD, assessed by 2D image technique such a DXA, is an estimate of the average amount of mineral per unit area in a section of bone facing the detector $(13,34)$. Since the hip strength indices assessed by AHA are estimated by use of the same mass distribution curve as BMD measurement, both CSA and CSMI depend of the amount of mineral present in the femoral neck, and consequently are not totally independent of BMD. Moreover, some data indicate that the usefulness of the hip strength analysis in addition to the BMD measurement for hip fracture prediction may be overestimated (13). In conclusion, our results do not confirm that increased hip fracture risk in type 1 diabetic men is mediated by the major decrease of BMD or hip strength indices and the contribution of diabetes-associated risk factors should be considered. These factors include increased risk of falling resulting from impaired vision in patients with advanced retinopathy or cataracts, poor balance, and orthostatic hypotension from peripheral and autonomic neuropathy, hypoglycemic episodes or limited mobility in patients with extensive neuropathy, foot ulcers or amputations. 


\section{References}

1 Faulkner KG. Improving femoral bone density measurements. Journal of Clinical Densitometry 20036 353-358.

2 Kayath MJ, Tavares EF, Dib SA \& Vieira JGH. Prospective bone mineral density evaluation in patients with insulin-dependent diabetes mellitus. Journal of Diabetes and its Complications 199812 133-139.

3 Krakauer JC, McKenna MJ, Buderer NF, Rao DS, Whitehouse FW \& Parfitt AM. Bone loss and turnover in diabetes. Diabetes $19954 \mathbf{4}$ 775-782.

4 Valerio G, del Puente A, Esposito-del Puente A, Buono P, Mozillo E \& Franzese A. The lumbar bone mineral density is affected by longterm poor metabolic control in adolescents with type 1 diabetes mellitus. Hormone Research 200258 266-272.

5 Liu EY, Wactawski-Wende J, Donahue RP, Dmochowski J, Hovey KM \& Quattrin T. Does low bone mineral density start in post-teenage years in women with type 1 diabetes? Diabetes Care $2003262365-2369$.

6 Salvatoni A, Mancassola G, Biasoli R, Cardani R, Salvatore S, Broggini M \& Nespoli L. Bone mineral density in diabetic children and adolescents: a follow-up study. Bone 200434 900-904.

7 Nicodemus KK \& Folsom AR. Type 1 and type 2 diabetes and incident hip fractures in postmenopausal women. Diabetes Care $2001241192-1197$.

8 Forsén L. Diabetes mellitus and the incidence of hip fracture: results from the Nord-Trondelag Health Survey. Diabetologia 1999 42 920-925.

9 Ahmed LA, Joakimsen RM, Berntsen GK, Fønnebø V \& Schirmer H. Diabetes mellitus and the risk of non-vertebral fracture: the Tromsø study. Osteoporosis International $2006 \mathbf{1 7}$ 495-500.

10 Bouillon R. Diabetic bone disease. Calcified Tissue International $199149155-160$.

11 Miao J, Brismar K, Nyren O, Ugarph-Morawski A \& Ye W. Elevated hip fracture risk in type 1 diabetic patients: a population-based cohort study in Sweden. Diabetes Care 200528 2850-2855.

12 Crabtree NJ, Kroger H, Martin A, Pols HAP, Lorenc R, Stepan JJ, Falch JA, Miazgowski T, Grazio S, Raptou P, Adams J, Collings A, Khaw K-T, Rushton N, Lunt M, Dixon AK \& Reeve J. Improving risk assessment: hip geometry, bone mineral distribution and bone strength in hip fractures and controls. The EPOS Study. Osteoporosis International 200213 48-54.

13 Ahlborg HG, Nguyen ND, Nguyen TV, Center JR \& Eisman JA. Contribution of hip strength indices to hip fracture risk in elderly men and women. Journal of Bone and Mineral Research $2005 \mathbf{1 0}$ 1820-1827.

14 Faulkner KG, Cummings SR, Black D, Palermo L, Gluer CC \& Genant HK. Simple measurement of femoral geometry predicts hip fracture: the study of osteoporotic fractures. Journal of Bone and Mineral Research 19938 1211-1217.

15 Gómez Alonso C, Díaz Curiel M, Hawkins Carranza F, Pérez Cano R \& Díez Pérez A. Femoral bone mineral density, neck-shaft angle and mean femoral neck width as predictors of hip fracture in men and women. Osteoporosis International 20008 714-720.

16 Beck TJ, Looker AC, Ruff CB, Sievanen H \& Wahner HW. Structural trends in the aging femoral neck and proximal shaft: analysis of the Third National Health and Nutrition Examination Survey dual-energy X-ray absorptiometry data. Journal of Bone and Mineral Research 200015 2297-2304.

17 Nelson DA, Barondess DA, Hendrix SL \& Beck TJ. Cross-sectional geometry, bone strength, and bone mass in the proximal femur in black and white postmenopausal women. Journal of Bone and Mineral Research 200015 1992-1997.
18 American Diabetes Association. Diagnosis and classification of diabetes mellitus. Diabetes Care 200629 S43-S48.

19 American Diabetes Association. Standards of medical care in diabetes-2006. Diabetes Care 200629 (Suppl) S4-S42.

20 Yoshikawa T, Turner CH, Peacock M, Slemenda CW, Weaver CM, Teegarden D, Markwardt P \& Burr DB. Geometric structure of the femoral neck measured using dual-energy X-ray absorptiometry. Journal of Bone and Mineral Research 19949 1053-1064.

21 World Human Organization. Assessment of fracture risk and its application to screening for postmenopausal osteoporosis: report of a WHO study group. WHO Technical report series No. 843, Geneva, World Health Organization, 1994, 1-129.

22 Lunt H, Florkowski CM, Cundy T, Kendall D, Brown LJ, Elliot JR, Wells JE \& Turner JG. A population-based study of bone mineral density in women with longstanding type 1 (insulin dependent) diabetes. Diabetes Research and Clinical Practice 199840 31-38.

23 Kayath MJ, Tavares EF, Dib SA \& Vieira JGH. Prospective bone mineral density evaluation in patients with insulin-dependent diabetes mellitus. Journal of Diabetes and its Complications 199812 133-139.

24 Ponder SW, McCarminck DP, Fawcett HD, Tran AD, Ogelsby GW, Bronhard BH \& Travis LB. Bone mineral density of the lumbar vertebrae in children and adolescents with insulin-dependent diabetes mellitus. Journal of Pediatrics 1992120 541-545.

25 Gallacher SJ, Fenner JA, Fisher BM, Quin JD, Fraser WD, Logue FC, Cowan RA, Boyle IT \& MacCuish AC. An evaluation of bone density and turnover in premenopausal women with type 1 diabetes mellitus. Diabetic Medicine 199310 129-133.

26 Bridges MJ, Moochhala SH, Barbour J \& Kelly CA. Influence of diabetes on peripheral bone mineral density in men: a controlled study. Acta Diabetologica 200542 82-86.

27 De Schepper J, Smitz J, Rosseneu S, Bollen P \& Louis O. Lumbar spine bone mineral density in diabetic children with recent onset. Hormone Research 199850 193-196.

28 Thrailkill KM, Lumpkin CK, Bunn RC, Kemp RC \& Fowlkes JL. Is insulin an anabolic agent in bone? Dissecting the diabetic bone for clues American Journal of Physiology, Endocrinology and Metabolism 2005289 E735-E745.

29 McCabe LR \& Botolin S. Osmoadaptation impairs osteoblast differentiation. Journal of Bone and Mineral Research 200318 (Suppl) S229.

30 Jehle PM, Jehle DR, Mohan S \& Bohm BO. Serum levels of insulinlike growth factor system components and relationship to bone metabolism in type I and type II diabetes mellitus patients. Journal of Endocrinology 1998159 297-306.

31 McCarthy AD, Uemura T, Etcheverry SB \& Cortizo AM. Advanced glycation endproducts interfere with integrin-mediated osteoblastic attachment to type-I collagen matrix. International Journal of Biochemistry and Cell Biology 200436 840-848.

32 Burkhart R, Moser W, Bartl R \& Mahl G. Is diabetic osteoporosis due to microangiopahy? Lancet 198211844.

33 Duboef F, Hans D, Schott AM, Kotzki PO, Favier F, Marcelli C, Meunier PD \& Delmas PD. Different morphometric and densitometric parameters predict cervical and trochanteric hip fracture: the EPIDOS Study. Journal of Bone and Mineral Research 199712 1895-1902.

34 Nilsson BE, Johnell O \& Peterson C. In vivo bone-mineral measurement. How and why - a review. Acta Orthopoedica Scandinavica $199061275-281$.

Received 4 September 2006

Accepted 5 October 2006 